\title{
Visualizing physiological signals in real-time
}

\author{
Sebastián Sepúlveda ${ }^{\ddagger *}$, Pablo Reyes ${ }^{\ddagger}$, Alejandro Weinstein ${ }^{\ddagger}$ \\ https://www. youtube. com/watch?v=6WxkOeTuX7w
}

\begin{abstract}
This article presents an open-source Python software package, dubbed RTGraph, to visualize, process and record physiological signals (electrocardiography, electromyography, etc.) in real-time. RTGraph has a multiprocess architecture. This allows RTGraph to take advantage of multiple cores and to be able to handle data rates typically encountered during the acquisition and processing of biomedical signals. It also allows RTGraph to have a clean separation between the communication and visualization code. The paper presents the architecture and some programming details of RTGraph. It also includes three examples where RTGraph was adapted to work with (i) signals from a Inertial Measurement Unit (IMU) in the context of a biomechanical experiment; (ii) electromyography signals to estimate muscle fatigue; and (iii) pressure signals from a device used to monitor nutrition disorders in premature infants.
\end{abstract}

Index Terms-real-time processing, visualization, signal processing

\section{Introduction}

A common task in biomedical research is to record and visualize physiological signals in real-time. Although there are several options to do this, they are commonly based on proprietary tools, associated with a particular signal acquisition device vendor. This article presents RTGraph, an open-source software package (under MIT license) written in Python, to visualize and record physiological signals in real-time, such as electrocardiography, electromyography and human movement. RTGraph is also capable of doing real-time processing, such as filtering and spectral estimation. RTGraph is open-source, ${ }^{1}$ extensible, and has been tested on different Linux distributions, including the RaspberryPi (ARM architecture). RTGraph has a modular design, with a clear separation among its different functionalities, making it easy to add new signal processing tasks, to use different communication protocols (serial, Bluetooth, Sockets, etc.), and customize the user interface for the specific needs of the application.

The main aim of RTGraph is to display multiple signals in realtime and to export them to a file. In the current implementation, the communication between RTGraph and the acquisition device is through the serial port, and it is implemented using the PySerial library. Other communication protocols can be easily added. The real-time display of the signals is implemented using the PyQtGraph library. ${ }^{2}$ RTGraph has a multiprocess architecture, based

\footnotetext{
* Corresponding author: ssepulveda.sm@gmail.com

¥ Escuela de Ingeniería Civil Biomédica, Facultad de Ingeniería, Universidad de Valparaíso
}

Copyright $@ 2015$ Sebastián Sepúlveda et al. This is an open-access article distributed under the terms of the Creative Commons Attribution License, which permits unrestricted use, distribution, and reproduction in any medium, provided the original author and source are credited.

1. Available at https://github.com/ssepulveda/RTGraph. on the multiprocessing Python standard library. This allows having concurrent processes for receiving, processing, and displaying the data. Signal processing tasks, such as spectral estimation, are based on the SciPy/NumPy stack [Ste11]. This architecture makes it possible to ensure that no data is lost and that the user interface has a fast response.

\section{Software architecture}

The applications described in this article can be classified as a "data logger". A data logger needs to acquire a stream of data, add a time stamp to the data (if required), and export the timestamped data to a file in a known file format, such as comma separated value (CSV) format. Optionally, the application can do some processing (filtering, spectral estimation, etc.) before saving the data. In addition, it is also useful to be able to visualize, in real-time, the stream of data.

When developing, evaluating, or validating new hardware or software, it is important to control the outcome of the algorithms and the fidelity and performance of the data acquisition process. In particular, in the field of Biomedical Engineering, the acquisition and processing of biological signals need to be reliable and with a tight control over the sampling frequency. It is also fundamental to ensure that no data is lost during the acquisition and logging process. From a practical point of view, having to wait for the data to be stored before visualizing it (possibly in another program) is cumbersome, slowing down the development process. For these reasons, in this article we present a program capable of: receiving data from a variety of sources (serial port, Bluetooth, Zigbee, Sockets, etc.); processing and visualizing the data in real-time; and saving the data in a file.

The first version of this program was developed for biomechanical engineering research. In our case, this research involves logging, processing and the display in real-time of the signals generated by a nine degrees of freedom inertial measurement unit (9DOF-IMU) [Roe06]. This requires acquiring nine signals with a sampling rate of at least $100 \mathrm{~Hz}$. Six additional signals are computed through a sensor fusion algorithm [Mad11]. A total of 15 signals are displayed and exported as a CSV file. We designed the architecture of the program with these requirements in mind.

\section{Real-time graphics library}

Real-time visualization is a key component of our program. To satisfy our requirements we needed a fast and portable graphics library. Since we implemented the GUI in PyQT, we also required that the graphics library should be embeddable in this framework.

2. Available at http://www.pyqtgraph.org. 
We used Matplotlib [Hun20] in the first version of the program. This option worked out of the box. We were able to embed a Matplotlib plot in the GUI and interact with it through other elements of the UI without major complications. Although this approach worked for displaying one signal with a sampling rate of $30 \mathrm{~Hz}$, we started to notice a degradation on performance as we increased the number of signals. It is important to note that this is not a flaw of Matplotlib, since the main focus of the library is the production of publication of quality figures, and not the display of real-time data.

Next, we tried PyQtGraph [Cam15]. It is a pure Python implementation, with a focus on speed, portability and a rich set of features. Unlike Matplotlib, PyQtGraph is designed to do realtime plotting and interactive image analysis. It is built on top of PyQt4/PySide, giving easy integration and full compatibility with the Qt framework. This allows using tools like Qt Designer to design the GUI. Using Qt Designer and the examples provided with the PyQtGraph library, it is easy to configure and customize the widgets. PyQtGraph is also built on top of NumPy, facilitating and improving the performance of the manipulation of numerical data. In addition, PyQtGraph wraps up some NumPy/SciPy signal processing functions such as the Fast Fourier Transform and some linear and non-linear filters. ${ }^{3}$

\section{Threading versus Multiprocessing}

After using PyQtGraph to its limits in a multithreaded architecture, we could not reliably achieve the desired performance. The limitations of threads in Python [Bea10] combined with the interaction between the UI (main thread) and communication thread, resulted in data losses when the data rate was too high. The Global Interpreter Lock (GIL) [Bea10] prevents threads from taking advantage of multicore systems. In short, it means that a mutex controls threads access to memory. There are ways to work around this limitation. For instance, many of the NumPy primitives take advantage of multiple cores. ${ }^{4}$ However, in our case we need to parallelize the reception of the data, the visualization, the processing, and the logging.

To overcome the GIL limitations we used the multiprocessing module, belonging to the Python Standard Library. This module provides an API similar to the threading module, but it uses subprocesses instead of threads [Pyt15]. By letting the OS control the subprocesses, it is possible to take advantage of the multiple cores available on the platform.

\section{Putting it all together}

After selecting the key components of the program, the remaining problem is to orchestrate the communication among the processes. We pay special attention to data synchronization, since there are specific considerations that should be taken into account when working with multiple processes.

Figure 1 shows the architecture of RTGraph. The architecture allow us to: (1) Have a multiplatform program; (2) have a separation between the reception and parsing of input data stream and

3. We also evaluated the PyQwt library (http://qwt.sourceforge.net/). This library provides a Python interface to the Qwt library. It is a light implementation with an easy QT integration. It is fast enough to support real-time display of the data. However, this library is not currently maintained, and its author recommended using PyQtGraph (see http://comments.gmane.org/gmane.comp. graphics.qwt.python/506).

4. See http://wiki.scipy.org/ParallelProgramming for details.

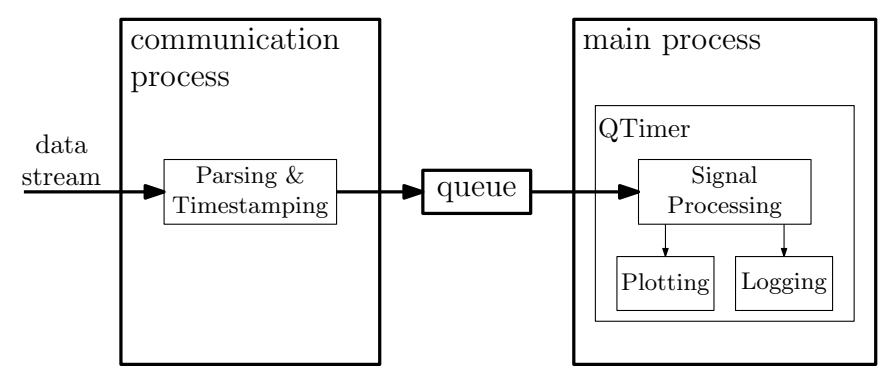

Fig. 1: Diagram of the software architecture. There are two independent processes. The communication process reads the incoming data stream, parses it, adds a time-stamp (if necessary), and puts the processed data into a queue. The main process reads the data from the queue, processes the data, and then updates the plot and logs the data to a file.

the plotting and logging tasks. The following is a description of each process.

1) Communication process: This process is responsible for receiving and parsing the data stream sent by the device. The implementation consists of an abstract class, that subclasses the Process class from the multiprocessing library. Therefore, the methods init__ and run are overwritten. We also added methods start and stop to properly start and stop the subprocesses. The class also has methods common to different communication protocols (serial, sockets, etc.). The details of each protocol are implemented in each subclass. This process is also responsible of validating the data and adding the time-stamp to the data, in case the device does not provide it. This guarantees that the data is always time-stamped.

2) Main process: The main process is responsible for initializing the different subprocesses and for coordinating the communication between them. As shown in figure 1, this process instantiates the components that will allow the communication between the subprocesses and also manage the different UI elements. A Queue, as implemented by the multiprocessing module, is used to connect the communication process with the main process. A Qt Timer is set to update the real-time plot. By updating the plot at a known frequency, we can control the responsiveness of the program under different conditions. Each time the QtTimer triggers a plot update (30 times per second), the queue is processed. The queue is read until it is empty and then the plot is updated.

Figure 2 shows the processes viewed by htop during the execution of the program. The first process (PID 3095) corresponds to the process initiated by the application. The second one is the communication process (PID 3109). ${ }^{5}$

\section{Programming details}

The template for the communication process is implemented through the CommunicationProcess class. This template allows for processing data streams coming from a variety of

5. By default htop shows the processes and threads together. Pressing the $\mathrm{H}$ key while the program is running shows or hides the threads. In figure 2 , the screen is configured to show only the processes. 
protocols (serial, sockets, bluetooth, etc.). The design of the class also allows changing some of the communication parameters during run-time. In addition, since the class inherits from the Process class, it is trivial to run several instances of the class to receive from multiple devices simultaneously. For instance, it is possible to instantiate the class twice to receive data form two different serial ports at the same time. The following code snippet shows the basic structure of the class.

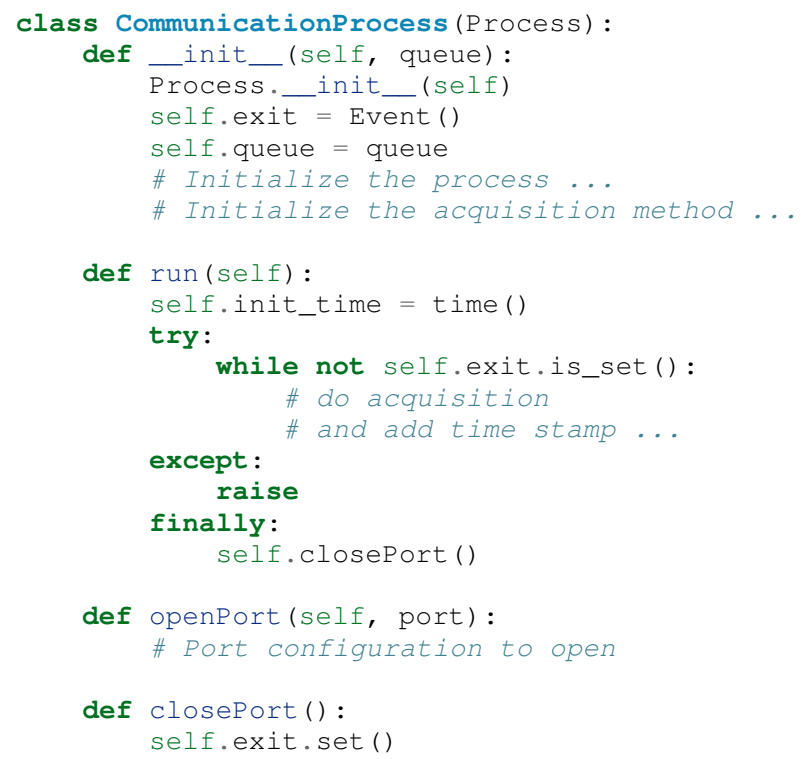

One of the key methods of the CommunicationProccess class is run. The following code snippets is an example of how to write a serial port interface.

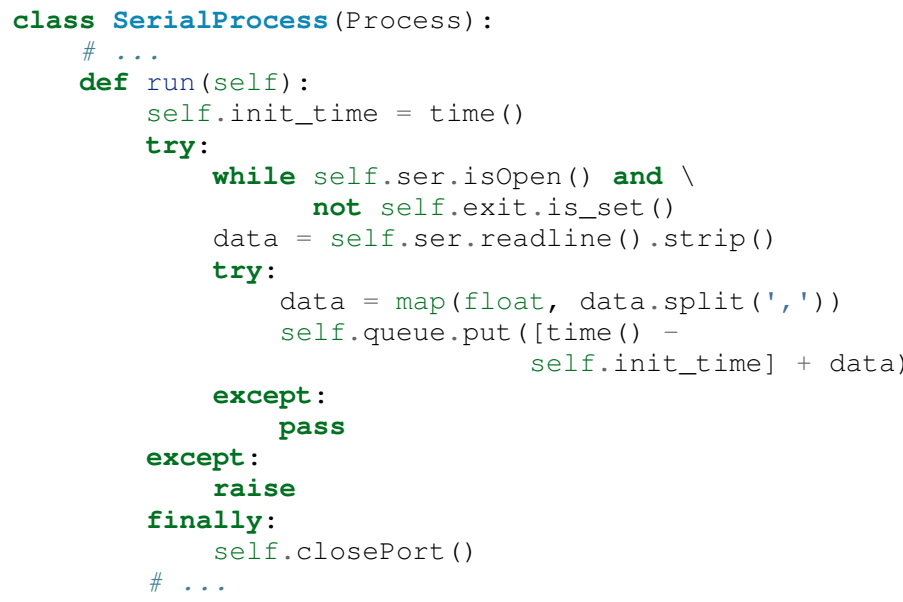

In this case, run computes the time stamp, then checks if the serial port is open and if the process is not exiting. If both statements are true, a line is read from the serial port. Then, the data is parsed (in this example, the data stream consists of CSV floats). Finally, if the data is valid it is placed in the queue.

The main process is implemented through the MainWindow class. It is a subclass of the QtGui. QMainWindow class. Inside this class we define the proper acquisition method (serial, sockets, bluetooth, etc.) and the basic plot configurations, and we configure the timers used to update the plots, which trigger the update_plot method. The following code snippet shows the basic structure of the class.

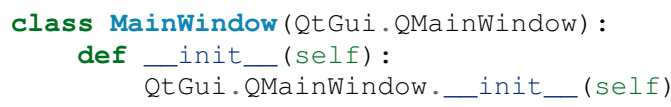

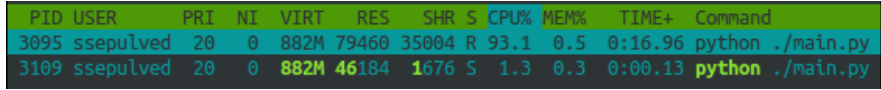

Fig. 2: Screenshot of htop showing the processes associated with the program. The first process (PID 3095) corresponds to the process initiated by the application. The second one is the communication process (PID 3109).

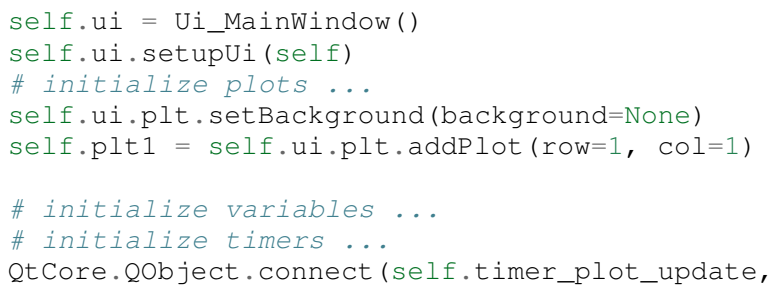

The start method initializes the communication process. This method is triggered every time the Start button is pressed. This allows to change the communication parameters (port name, bauds, etc.) during execution time.

The plot details are also defined in the MainWindow class. The following code snippets shows how to customize some PyQtGraph options, such as titles, labels, and line colors.

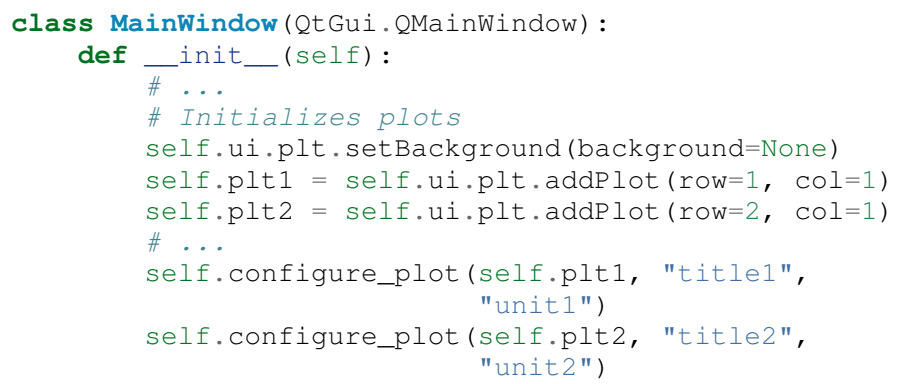




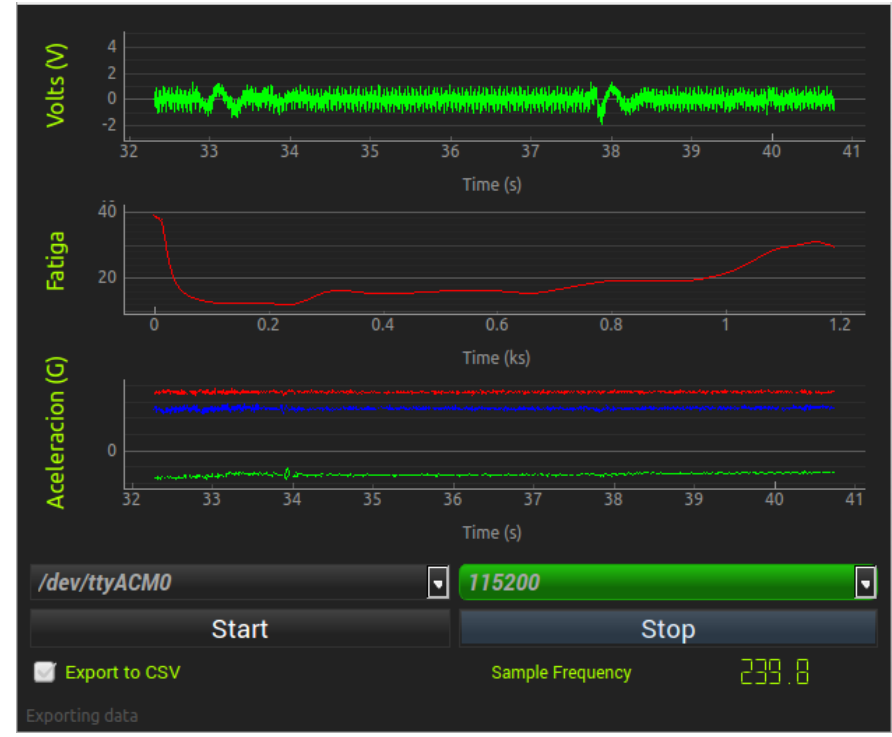

Fig. 3: Screenshot of RTGraph customized and modified to display 3 signals: an EMG signal (first panel), an estimation of the fatigue level (second panel) based on the acquired EMG signal, and three acceleration signals (third panel).

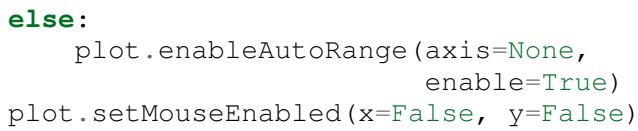

The class sets the layout of the plots through calls to self.ui.plt.addPlot methods. Then, each plot is configured by the configure_plot method, where details such as title, range, color, and font sizes are set.

\section{Results}

We have used RTGraph with a serial port data stream corresponding to a signal with a sampling frequency of $2 \mathrm{kHz}$. We have also used it with a data stream from a TCP/IP socket corresponding to 20 signals with a sampling frequency of $500 \mathrm{~Hz}$.

In a biomechanical study we used our program to evaluate a prototype of a wearable device used to estimate muscle fatigue through the EMG signal. RTGraph was customized to acquire and record these data. We also incorporated some steps of a fatigue estimation algorithm [Dim03] in the processing pipeline. We found that having real-time feedback of the signal simplified the procedure to position the wearable device correctly, drastically reducing the amount of time required by the experiments. Figure 3 shows a screenshot of the program while acquiring an EMG signal using a wearable device to study muscle fatigue. The figure shows an EMG signal (first panel), a real-time estimation of the fatigue level (second panel) based on the acquired EMG signal, and three acceleration signals (third panel). See the following links for a video of RTGraph being used to acquire these signals: https://www.youtube.com/watch?v=sdVygxpljII, https:// www.youtube.com/watch?v=6WxkOeTuX7w.

An important feature of our program is the ease with wich it can be customized to a specific application. For instance, RTGraph is being used to acquire a set of pressure signals from a device (as seen in figure 4) used to monitor nutrition disorders in premature infants. The customization included: (1) modifying RTGraph to acquire two pressure signals using bluetooth; and (2) to perform

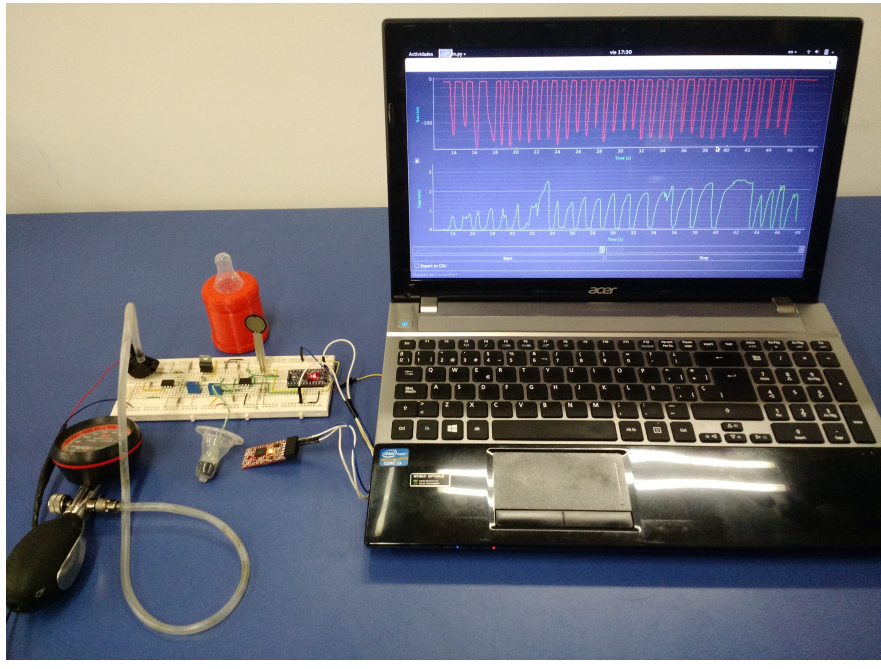

Fig. 4: Photo of the prototype device used to monitor nutrition disorders in premature infants. An Arduino development platform is used to acquire the signals (two pressure measurements). These signals are acquired by a computer running a modified version of RTGraph.

some specific signal processing before the visualization. In this example it is important to emphasize that the changes to the program were made by a researcher other than the main developer of our program. We claim that this is possible because our program is written in Python. This makes it easier to understand and modify the code compared to a program written in a lower-level language.

The software package presented in this article has been tested with different devices, communication protocols, platforms and operating systems (OSs). The initial development was done and tested on the platforms x86, x64 and ARM (RaspberryPy) running Linux. However, this version of RTGraph did not work as expected on OS X and Windows, due to some restrictions of the multiprocessing library in these OSs. Despite the fact that OS X is a Unixlike OS, there are some multiprocessing methods not implemented in the multiprocessing library. In particular, the method qsize, used to get the approximate size of the queue, is not implemented in OS X. The lack of the os.fork () call in Windows adds some extra limitations when running a program on this OS. Since in this case a child process can not access the parent resources, it is necessary that subclasses of the Process class must be picklable. Although the documentation of the library contains some suggestions to overcome these restrictions, currently we are not able to run our program on Windows.

\section{Conclusions}

In this article we presented a program developed to record, process and visualize physiological signals in real-time. Although many people consider Python as a "slow" language, this article shows that it is possible to use Python to write applications able to work in real-time. At the same time, the clarity and simplicity of Python allowed us to end up with a program that it is easy to modify and extend, even by people who are not familiar with the base code.

We also believe that our solution is a contribution to the open-source and Do It Yourself (DIY) communities. Typically, programs to receive and manipulate data in real-time are developed using proprietary tools such as LabView or MATLAB. The cost of these tools denies members of these communities access to 
solutions like those described in this article. As we showed in the results section, in many cases we have used the program with an Arduino acting as an acquisition device. This is a common situation, and we believe that our program can be extended to be used in other fields in need of similar tools.

In the future our first priority is to make our program work on platforms running OS X and Windows. We are currently investigating how to overcome the restriction imposed by the multiprocessing platform on these OSs. Next, we will focus on improving the UI. In particular, we will add an option to change some plotting and processing parameters on the fly, instead of requiring a change in the source code. Finally, we will refactor the architecture of the program to improve the performance, so we can handle higher data rates. In this respect, the main change we plan to do is to move the signal processing computation to another process, leveraging the existence of multi-core machines.

\section{Acknowledgments}

This research was partially supported by the Advanced Center for Electrical and Electronic Engineering, Basal Project FB0008, Conicyt.

\section{References}

[Bea10] D. Beazley. Understanding the Python GIL, In PyCON Python Conference. Atlanta, Georgia, 2010.

[Cam15] L. Campagnola. PyQtGraph. Scientific Graphics and GUI Library for Python, http://www.pyqtgraph.org/

[Dim03] N. Dimitrova and G. Dimitrov. Interpretation of EMG changes with fatigue: facts, pitfalls, and fallacies. Journal of Electromyography and Kinesiology 13.1 (2003): 13-36.

[Hun20] J. D. Hunter. Matplotlib: A 2D graphics environment, Computing In Science \& Engineering, 9(3):90-95, IEEE COMPUTER SOC, 2007.

[Mad11] S. Madgwick, Andrew JL Harrison, and Ravi Vaidyanathan. Estimation of IMU and MARG orientation using a gradient descent algorithm., Rehabilitation Robotics (ICORR), 2011 IEEE International Conference on. IEEE, 2011.

[Pyt15] Python Software Foundation, 16.6 multiprocessing - Processbased "threading" interface, https://docs.python.org/2/library/ multiprocessing.html

[Roe06] D. Roetenberg, Inertial and magnetic sensing of human motion. University of Twente, 2006.

[Ste11] S. van der Walt, S.C. Colbert and G. Varoquaux, The NumPy Array: A Structure for Efficient Numerical Computation, Computing in Science \& Engineering, 13, 22-30, 2011. 